\title{
Design for Additive Manufacturing, to produce assembled products, by SLS
}

\author{
Nicolae Bâlc ${ }^{1, *}$, and Cristian Vilău ${ }^{1}$ \\ ${ }^{1}$ Technical University of Cluj-Napoca, B-dul. Muncii, No. 103-105, Cluj-Napoca, Romania
}

\begin{abstract}
The paper presents a new methodology on how to design a product, which could be produced directly as an assembly, instead of manufacturing all individual parts, to be assembled after words, in a classical manner. A detailed case study is presented, on how the product's structure could be simplified, the number of the components being drastically reduced. The theoretical Design for Assembly (DFA) methodology was tested and validated, by manufacturing the physical products, using the Selective Laser Sintering (SLS) machine.
\end{abstract}

\section{Introduction}

The Additive Manufacturing (AM) technologies bring more alternatives both on design and manufacturing the products, which can be obtained directly assembled, without being necessary to produce separate parts and to assemble all of them later on, in a classical manner. It is not only that the assembly time and costs could be saved, but there are also other advantages, such as to simplify the structure of the product and reduce the number of the components, by eliminating the assembly elements.

(a)

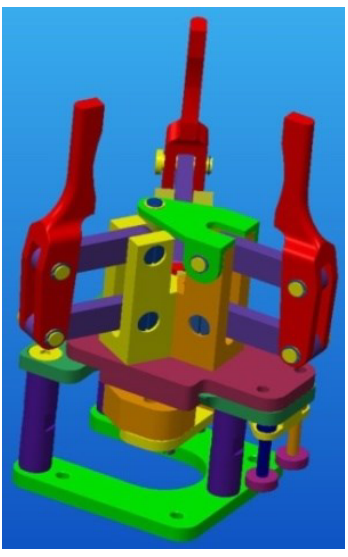

(b)

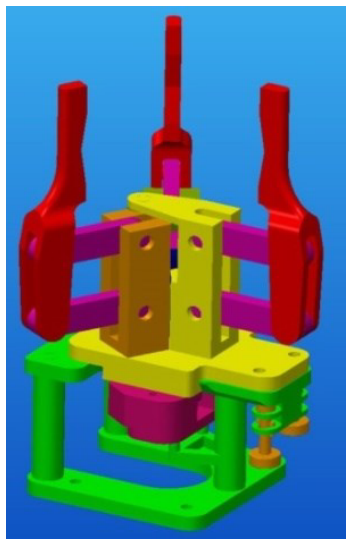

Fig. 1. Design for manufacturing assembled products, versus classical design.

\footnotetext{
* Corresponding author: nicolae.balc@tcm.utcluj.ro
} 
Selective Laser Sintering (SLS) is a suitable technology to produce assembled products, as it uses powder materials, which could be eliminated easy at the end of the SLS process. The SLS process takes place in a solid faze, so the excess of powder could be removed during post-processing, if the assembly has been properly designed, with suitable gaps, in between the moving elements.

A special gripper, for a Kuka robot, was designed as part of the $\mathrm{PhD}$ thesis, in order to be flexible and able to handle both, rotational and prismatic parts [3]. This new gripper, illustrated in figure 1a, is composed by 61 elements, if the parts are to be made separately and being assembled at a later stage. If this gripper can be made by SLS, it's structure could became a lot simpler, and the new gripper could be designed for AM, the number of the components being decreased down to 19 , as illustrated in figure $1 \mathrm{~b}$.

\section{Design to manufacture assembled products}

It is a concurrent engineering, as the authors paid attention in the same time, to the functionality of the gripper and to the new possibilities of the SLS manufacturing technology, in order to redesign the gripper, to be made by SLS, directly assembled. We managed to remove more than 2 thirds of the components, as the screws, nuts, pins, elastic rings and other assembly elements, are not necessary any more.

The functionality of the new design of the gripper should remain exactly the same, as the version of the gripper, designed in a classical manner. The base frame is going to be fixed onto the pneumatic linear motor. Besides of this frame, most of the other parts are mobile, each of them having a complex functionality purpose. The gripper is meant to be able to handle, both rotational and prismatic parts. That is why, many of its components need to adapt their position, from 2 to 3 jaws and back to 2 .

Design for AM and virtual assembly require detailed analyses of the functionality of the new product [2]. For each component and/or assembly element which is a candidate for elimination, the designer needs to change the adjacent components of the product, in order to undertake the role of the eliminated parts.

The authors evaluated each component of the classical version of the gripper and the following three basic Design for Assembly (DFA) criteria were applied, in order to check if a part is a candidate for elimination [1]:

- Is this part moving (rotational or linear), with respects to the adjacent parts?

- Does this part have to be made from a different material, as compare to the adjacent parts?

- Does this part have to be separate, in order to be able to assemble or disassemble other parts?

According to the Boothroyd \& Dewhurst methodology, if the answer is NO, to all the upper 3 questions, this part is a candidate for elimination. If the answer is YES to at least one of these 3 questions, then the analysed part has to remain separate.

The linear movement from the step motor is taken by the bracket, which is acting all 3 racks at once. Each of the racks is acting one of the 3 arms of the gripper. The rack is having a linear movement and it rotates the pinion (which has a gear sector on one side). An intermediary element with two cylindrical joints was designed, suitable for a maximum $65^{\circ}$ rotating angle. The distance between the holes is depending of the maximum opening diameter, imposed to the gripper. The movement of the jaws is a parallel translation movement, obtained from the rotation of the pinion, around its primary cylindrical joint. The jaw is supported and guided by the intermediary element, connected onto the jaw by a cylindrical joint. 
One of the 3 jaws has the supporting joint, fixed onto the central raceway of the gripper, which has a complex shape and belongs to the base plate. The other 2 jaws have intermediary elements fixed on special supports. Switching the gripper from 3 jaws gripping of the rotational parts, to 2 jaws gripping of the prismatic parts, is done manually, by linear transversal movement of the intermediary plate, which rotate the vertical supports, to align the 2 jaws.

\section{Virtual assembly the new product, designed for AM}

The "Creo Parametric" software was used to design the product to be made as an assembly, directly. Each of the 19 components of the new simplified structure of the gripper was designed in a separate part file. The first component called support is considered implicitly fixed, connected onto the pneumatic motor.

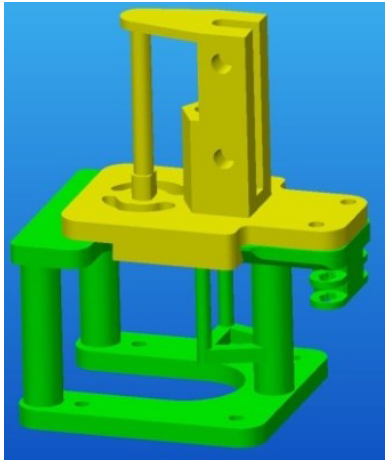

Fig. 2. DFA Considerations.

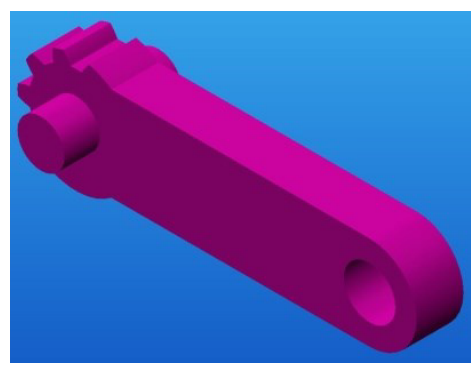

Fig. 3. Redesigned pinion.

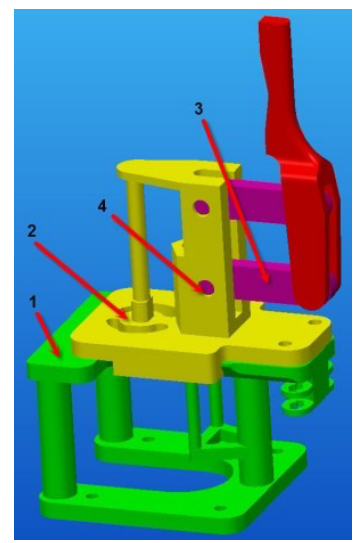

Fig. 4. The first jaw, assembled.

For the next component, which is the intermediary plate, the linear movement conditions were set up, to be acted manually. The length of the translation movement is correlated with the maximum allowed movement, from 3 jaws gripping to 2 jaws gripping.

The pinion illustrated in figure 3 comes next into the assembly and here the novelty and the difference appear, as compare to the classical procedure, when the parts were assembled one by one. Within the design for AM, the authors designed the pin, as being permanently joint (connected) to the pinion, so that no any separate bar is necessary anymore, for that cylindrical joint. Up to now, such an assembly could have been produced virtually, only. The new AM technologies open new doors and now such a virtual assembly could also be practically produced by SLS.

The research and practical experiments undertaken by the authors, allowed to find out the practical conditions to produce such an assembled product design. The gaps between the shaft and the surrounding support plate should be in accordance both, with the functional requirements and with the grain size and layer thickness of the SLS process. The gripper has been designed in two alternatives, with the space values of $0.1 \mathrm{~mm}$ and $0.2 \mathrm{~mm}$, in between all its moving elements.

Figure 4 illustrates how the first jaw was virtually assembled, for the product to be made by SLS. The rack was assembled next and in a similar manner, all the new designed components were virtually assembled. The rack has a cylindrical guiding surface (within the cylindrical inner surface of the intermediate support) and could do a maximum $4 \mathrm{~mm}$ linear movement. For the gap between these elements, the $0.2 \mathrm{~mm}$ aperture was adopted, as 
well. This rack is also virtually assembled, as in a classical manner it would be impossible to be inserted. The rack (figure 5) has two continuous contact surfaces, the cylindrical area for self guiding (1) where it gets the movement onto, from the intermediary plate and the other cylindrical zone (2), acted by the linear motor.

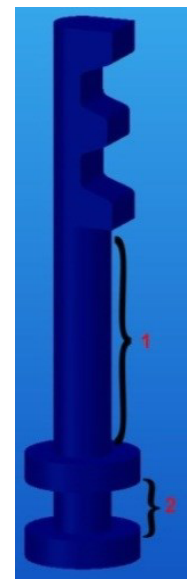

Fig. 5. The rack.

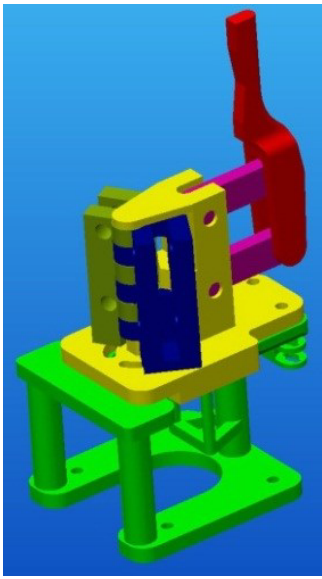

Fig 6. The joint supports.

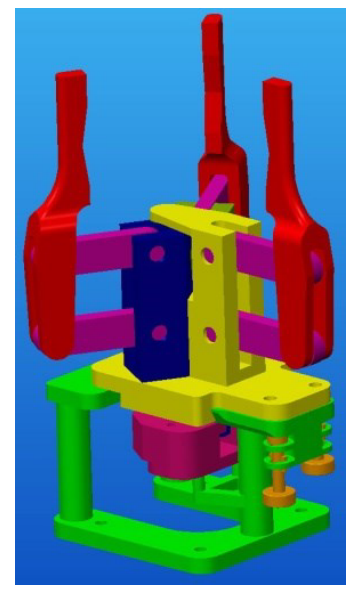

Fig. 7. All 19 parts virtually assembled.

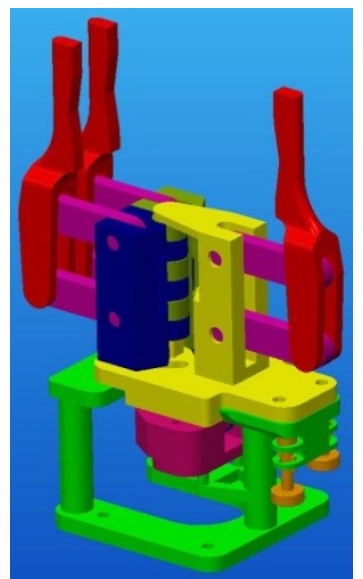

Fig. 8. Jaws for prismatic parts.

Two supports (green and blue - figure 6) were designed and assembled onto the same vertical shaft, with three intercalate zones, for cylindrical guiding. The rotation of these supports around their common shaft, is guided and limited by a cylindrical pin (which is joined onto the support), which is guided into a special slot, which was designed as a cavity into the intermediary plate. This slot has a special profile, representing the trajectory of the guiding element.

The intermediary elements of the other two jaws are added into assembly, so that the jaws could be assembled. Finally, the acting element and the two blocking bolts are assembled. Figure 7 illustrates all 19 components virtually assembled for cylindrical parts to be handled and figure 8 illustrates the gripper, when its jaws are aligned for prismatic parts to be handled.

\section{Selective Laser Sintering of the assembled products}

The practical case study was developed in order to test and validate the new DFA methodology developed by the authors. Two virtual assemblies designed with "Creo Parametric" software were saved as *.STL files and uploaded into the "Build set up" software of the SinterStation 2000 machine. Figure 9 illustrates the assemblies designed with $0.1 \mathrm{~mm}$ gap (left) and $0.2 \mathrm{~mm}$ gap (right). Both assemblies were further processed into the SLS machine's software (version 2.2.0.7). We also made experimental tests for higher gaps, such as $0.3 \mathrm{~mm}$, but in such cases we obtained a product with bad functionality. That gripper was blocking, because of the too big gaps between the moving elements.

The powder material (Duraform PA 650) used for the experimental research, has $50 \mu \mathrm{m}$ grain size. Both assemblies illustrated in fig. 9 were produced in the same time onto the Sinterstation 2000 SLS machine, available within the Department of Manufacturing Engineering, at Technical University of Cluj-Napoca.

The main technological parameters applied for the SLS process had the following values: laser power $4.5 \mathrm{~W}$, layer thickness $0.1 \mathrm{~mm}$, part bed temperature $170{ }^{\circ} \mathrm{C}$ and scanning speed $1.25 \mathrm{~m} / \mathrm{s}$. 
The AM techniques and in particular SLS have many advantages, such as the fact that the cost of the product does not increase with the complexity of the shapes to be made, but still require strong experience, to produce an accurate and reliable product. For this case study, the authors calibrated the SLS machine, using a standard part. Also, a set of scaling factors was applied, in order to compensate the shrinkage of the parts made by SLS.
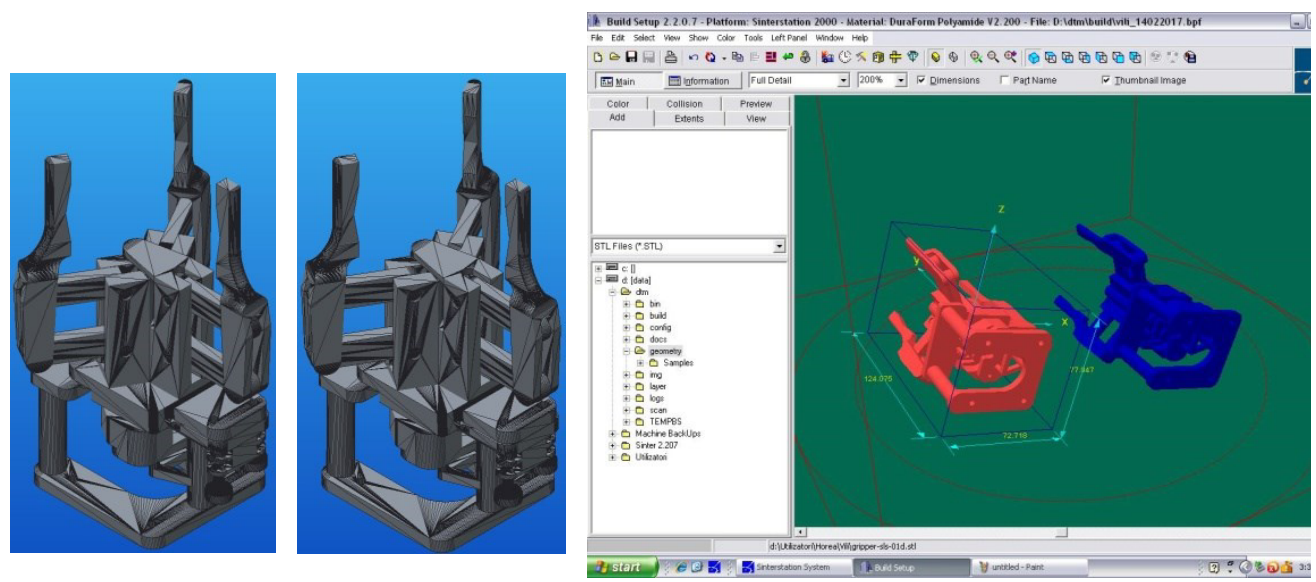

Fig. 9. Assemblies designed with 01. $\mathrm{mm}$ and $0.2 \mathrm{~mm}$ gaps.

Fig. 10. Optimized orientation for the SLS manufacturing, for the assembled products.

It was very important the position in which these 2 assemblies have been orientated within the working space of the SLS machine. If the gripper would have been built into the horizontal position, there would have been some advantages (such as short manufacturing time and theoretically a correct geometrical shape), but the disadvantage would have been, too much heat incorporated within the product, leading to significant shrinkage (3D deformations).

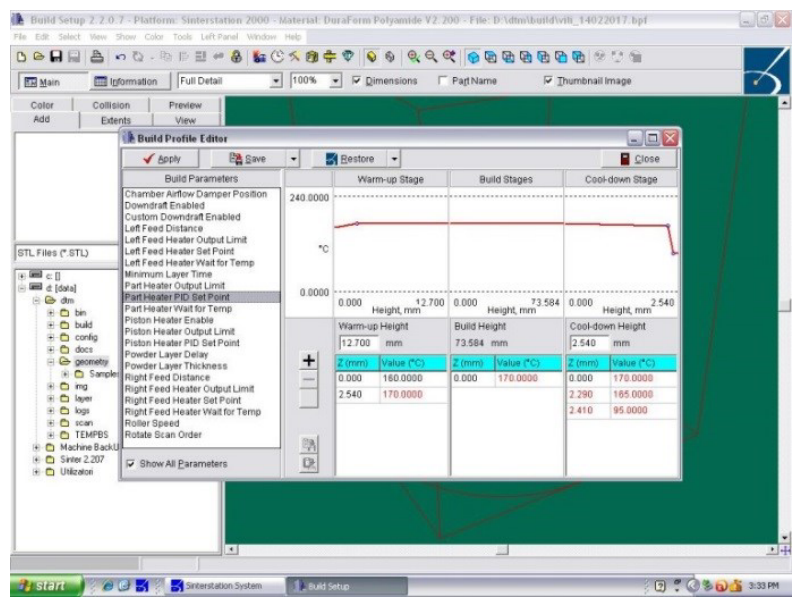

Fig. 11. Temperature cycles time, for SLS assemblies.

The authors found an optimized position of the gripper during its SLS manufacturing, at double angled position $\left(15^{\circ}\right.$ rotation about the $\mathrm{X}$ axes and $15^{\circ}$ rotation about the $\mathrm{Y}$ axes $)$. In this way, we made the assembled grippers by adding layers with different shapes, leading to smaller heat incorporated within the product, smaller shrinkage, so a higher accuracy of the SLS products. In this case, the disadvantage was a higher manufacturing 
time, which is proportional with the high of the part, but this increase of time is not causing inconvenience for such a small volume production.

The building time was approx. 9 hours for the two grippers illustrated in figure 12 , which were built together, in the same time, onto the SLS machine.
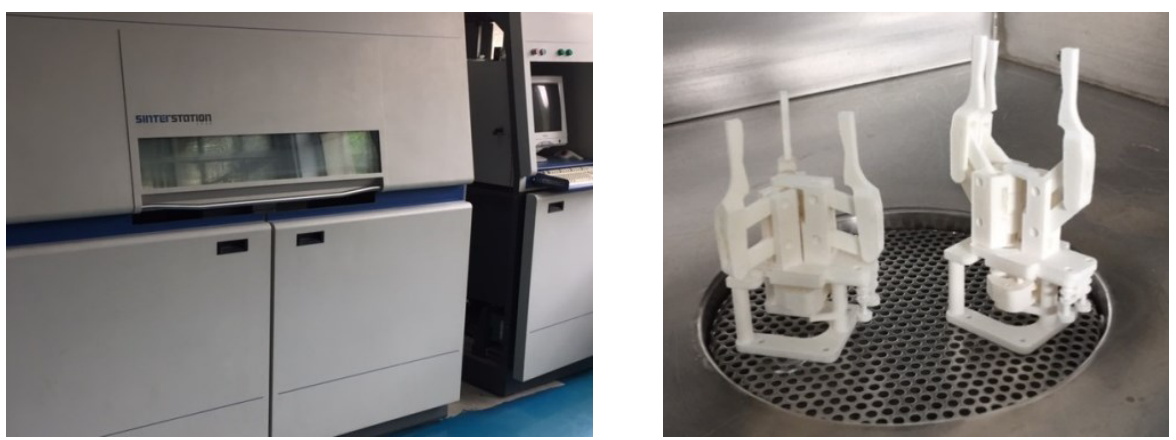

Fig. 12. Assembled products, made by SLS.

\section{Conclusions}

The new methodology on how to design a complex product, in order to be produced by SLS as an assembly, was tested and validated.

This practical case study shows both, the new possibilities and the advantages of the new design method. The number of components was decreased from 61 down to 19 , as only the functional components have to be incorporated and all the auxiliary and assembly elements could be eliminated.

Static and dynamic analyses were undertaken for this version of the DFA gripper, to check what the maximum gripping force would be, as compare to a similar gripper, made out of laminated steel, manufactured part by part and assembled in a classical manner.

Due to the significantly lower mechanical properties of this plastic material (PA 650), the maximum weight of a part which could be safely handled, is $0.5 \mathrm{~kg}$ (at the maximum velocity of the Kuka robot).

The research was supported within the PCCA PECIFCO project (No. 115/2014), H2020 AMaTUC project (GA 691787) and the OpTi-DeP project (No. 101BG/2016).

\section{References}

1. G. Boothroyd, P. Dewhurst, W. Knight, Product Design for Manufacture and Assembly, Third Edition ( CRC Press, USA, 2016)

2. S.B. Maidin, R.I. Campbell, E. Pei, Assembly Automation, 32 (3), 235-244 (2016)

3. Popan I.A., Balc N., Luca B., Popan A., Carean A, Applied Mechanics and Materials 808, 339$344,(2015)$

4. https://www.shapeways.com/tutorials/design_rules_for_3d printing

5. https://www.3dsystems.com/resources/sls-plastics-design-guide 